\title{
Some Results about the Isolated Calmness of a Mixed Variational Inequality Problem
}

\author{
Yali Zhao $\mathbb{D}^{1},{ }^{1}$ Hongying Li, ${ }^{2}$ Weiyi Qian, ${ }^{1}$ and Xiaodong Fan' \\ ${ }^{1}$ College of Mathematics and Physics, Bohai University, Jinzhou 121013, China \\ ${ }^{2}$ Department of Statistics, College of Arts and Science, University of Missouri-Columbia, Columbia, MO 65201, USA \\ Correspondence should be addressed to Yali Zhao; yalizhao2000@163.com
}

Received 20 January 2018; Accepted 7 March 2018; Published 8 April 2018

Academic Editor: Francesco Marotti de Sciarra

Copyright (c) 2018 Yali Zhao et al. This is an open access article distributed under the Creative Commons Attribution License, which permits unrestricted use, distribution, and reproduction in any medium, provided the original work is properly cited.

\begin{abstract}
It is well known that optimization problem model has many applications arising from matrix completion, image processing, statistical learning, economics, engineering sciences, and so on. And convex programming problem is closely related to variational inequality problem. The so-called alternative direction of multiplier method (ADMM) is an importance class of numerical methods for solving convex programming problem. When analyzing the rate of convergence of various ADMMs, an error bound condition is usually required. The error bound can be obtained when the isolated calmness of the inverse of the KKT mapping of the related problem holds at the given KKT point. This paper is to study the isolated calmness of the inverse KKT mapping onto the mixed variational inequality problem with nonlinear term defined by norm function and indicator function of a convex polyhedral set, respectively. We also consider the isolated calmness of the inverse KKT mapping onto classical variational inequality problem with equality and inequality constrains under strict Mangasarian-Fromovitz constraint qualification condition. The results obtained here are new and very interesting.
\end{abstract}

\section{Introduction}

In this paper, let us consider the following mixed variational inequality problem: find $x \in R^{n}$ such that

$$
\langle F(x), y-x\rangle+\phi(y)-\phi(x) \geq 0, \quad \forall y \in R^{n},
$$

where $F: R^{n} \rightarrow R^{n}$ is a twice continuously differentiable mapping and $\phi: R^{n} \rightarrow R \cup\{+\infty\}$ is a proper convex lower semicontinuous functional. Problem (1) is denoted by $\operatorname{MVIP}(F, \phi)$. Formulation of such problem was originally considered and studied by Brézis [1] in Hilbert space. It has been shown that a large class of obstacle, unilateral, contact, free, moving, and equilibrium problems arising in regional, physical, mathematical, engineering, and applied sciences can be studied in the unified and general framework of the mixed variational inequality problem. Some researchers considered and studied the existence of solutions and the numerical algorithms of the MVIP; for example, see [2] and the references therein.

On the other hand, many important optimization problems can be reformulated as the mixed variational inequality problem (1) to study. For example, if $F(x)=\nabla f(x)$, where $f$ : $R^{n} \rightarrow R$ is a continuously differentiable convex function, then (1) is related to the following convex optimization problem:

$$
\begin{aligned}
\min & f(x)+\phi(x) \\
\text { s.t. } & A x=b, \\
& g(x) \leq 0,
\end{aligned}
$$

where $f: R^{n} \rightarrow \bar{R}$ is a proper 1.s.c. Convex function, $g: R^{n} \rightarrow$ $R^{p}$ with $g=\left(g_{1}, \ldots, g_{p}\right)$ and each $g_{i}$ is a continuous convex function. Let us denote the feasible set of (2) by

$$
K=\left\{x \in R^{n}: A x=b, g(x) \leq 0\right\} .
$$

When there exists a vector $x_{0} \in R^{n}$ such that

$$
\begin{aligned}
A x_{0} & =b, \\
g\left(x_{0}\right)<0, & \\
& x_{0} \in \operatorname{ridom} \phi,
\end{aligned}
$$


then the optimality conditions for problem (2) coincide with the variational inequality (1). Indeed, problem (2) can be expressed as

$$
\min \hat{f}(x)=f(x)+\phi(x)+\delta_{K}(x),
$$

where $\delta_{K}$ is the indicator function of $K$. Obviously, $\widehat{f}$ is a proper lower semicontinuous convex function and if $\bar{x}$ is a solution to problem (2), then

$$
0 \in \partial \widehat{f}(\bar{x}) .
$$

Noting that

$$
x_{0} \in \operatorname{ridom} f \cap \operatorname{ridom} \phi \cap \operatorname{ridom} \delta_{K},
$$

we have from [3] that

$$
\partial \widehat{f}(\bar{x})=\nabla f(\bar{x})+\partial \phi(\bar{x})+N_{K}(\bar{x}) .
$$

Noting that $\partial\left[\phi+\delta_{K}\right](\bar{x})=\partial \phi(\bar{x})+N_{K}(\bar{x})$, we obtain from (6) and (8) that

$$
-F(\bar{x}) \in \partial\left[\phi+\delta_{K}\right](\bar{x}),
$$

which implies from the definition of subdifferential that

$$
\begin{aligned}
0 \leq & \langle F(\bar{x}), y-\bar{x}\rangle+\left[\phi(y)+\delta_{K}(y)\right] \\
& -\left[\phi(\bar{x})+\delta_{K}(\bar{x})\right],
\end{aligned}
$$

and this is an alternative expression for inequality (1) when $x=\bar{x}$.

It is well known that optimization problem model (2) has many applications arising from matrix completion, image processing, and statistical learning. The so-called alternative direction of multiplier method (ADMM) is an importance class of numerical methods for solving problem (2). When analyzing the rate of convergence of various ADMMs, an error bound condition is usually required; see Han and Yuan (2013) [4], Hong and Luo (2017) [5], and Han et al. (2015) [6]. The error bound can be obtained when the isolated calmness of the inverse of the KKT mapping bolds at the given KKT point; see Han et al. [6] and this observation motivates us to study the stability of problem (1).

The stability on variational inequalities is an important topic in optimization theory. For nonlinear programming, the strong regularity of the Karush-Kuhn-Tucker (KKT) system is completely characterized by Robinson (1980) [7] and Jongen et al. (1990) [8]. The equivalence of the strong regularity with the Aubin property for affine variational inequalities over convex polyhedral sets was established by Dontchev and Rockafellar (1996) [9]. Recently, results have been obtained for some matrix optimization problems. For instance, Y. Zhang and L. Zhang [10] proved the isolated calmness of the KKT mapping under the strict Robinson constraint qualification (SRCQ) and the second-order sufficient optimality condition for the nonlinear SDP problem. Han et al. [6] gave a characterization of isolated calmness of the KKT mapping for the convex composite quadratic semidefinite programming problems. Liu and Pan [11] extended the results in $[6,10]$ to the isolated calmness of the KKT mapping for the matrix optimization problem involving the epigraph cone of Ky Fan $k$-norm. Ding et al. [12] showed for a class of conic programming problems, under the Robinson constraint qualification, that the KKT solution mapping is robustly isolated calm if and only if both the strict Robinson constraint qualification and the second-order sufficient condition hold.

Recently, Mordukhovich and Sarabi (2017) [13] established a set of conditions to characterize the robust isolated calmness of the KKT mapping for a type composite optimization problem; in particular, they adopted noncritical multiplier to characterize the isolated calmness. In our paper, the problem considered is a variational inequality, not a composite optimization problem; and we use second-order sufficient optimality conditions and the strict Robinson constraint qualification to ensure the isolated calmness of the solution mapping. In addition, we mainly discuss the isolated calmness of the inverse KKT mapping on problem (1) with nonlinear term $\phi$ defined by norm function and the indicator function of a convex polyhedral set, respectively. And we also consider the isolated calmness of the inverse KKT mapping on classical variational inequality problem with equality and inequality constrains under strict Mangasarian-Fromovitz constraint qualification condition.

\section{Preliminaries}

In this section, we first recall some related definitions and lemma, which are needed in the subsequent discussions.

Let $Y$ be a finite dimensional real Euclidean space and $S$ : $R^{n} \Rightarrow Y$ be a set-valued mapping and $(\bar{x}, \bar{y}) \in \operatorname{gph} S$.

Definition 1. The set-valued mapping $S$ is said to be isolated calm at $\bar{x}$ for $\bar{y}$ if there exist a constant $k>0$ and neighborhoods $U$ of $\bar{x}$ and $V$ of $\bar{y}$ such that

$$
S(x) \cap V \subset\{\bar{y}\}+k\|z-\bar{z}\| B y \quad \forall x \in U,
$$

where $B y \subseteq Y$ is the unit ball in $Y$.

The graphical derivative is a convenient tool for investigating the isolated calmness property.

Definition 2 (see [14]). The graphical derivative of $S$ at $\bar{x}$ for $\bar{y}$ is a set-valued mapping $\operatorname{DS}(\bar{x} \mid \bar{y}): R^{n} \Rightarrow Y$ defined by

$$
\operatorname{DS}(\bar{x} \mid \bar{y})(u):=\left\{v \in Y \mid(u, v) \in T_{\mathrm{gphS}}(\bar{x}, \bar{y})\right\} .
$$

In the proof of the main theorem about the isolated calmness, we use the following basic characterization of the isolated calmness of the set-valued mapping $S$ at $\bar{x}$ for $\bar{y}$.

Lemma 3 (King and Rockafellar [15], Levy [16]). Let $(\bar{x}, \bar{y}) \epsilon$ gph $S$. Then $S$ is isolated calm at $\bar{x}$ for $\bar{y}$ if and only if $\{0\}=$ $\operatorname{DS}(\bar{x} \mid \bar{y})(0)$.

\section{The Isolated Calmness of MVIP}

In this section, we consider $\operatorname{MVIP}(F, \phi)(1)$, where $F: R^{n} \rightarrow$ $R^{n}$ is a continuously differentiable mapping and $\phi: R^{n} \rightarrow \bar{R}$ 
is a proper, lower semicontinuous and convex function. It is easy to know that $\operatorname{MVIP}(F, \phi)$ (1) is equivalent to the following inclusion:

$$
0 \in F(x)+\partial \phi(x) .
$$

For an extended real-valued function $f$, the Moreau-Yosida regularization of $f$, denoted by $e_{\lambda} f$, is defined by

$$
e_{\lambda} f(x)=\inf _{z}\left\{f(z)+\frac{1}{2 \lambda}\|z-x\|^{2}\right\} .
$$

It follows from [14] that, when $f$ is a proper lower semicontinuous convex function, $e_{\lambda} f$ is continuous differentiable and $\nabla e_{\lambda} f(x)=(1 / \lambda)\left[x-p_{\lambda} f(x)\right]$, where $p_{\lambda} f$ is the proximal mapping of $f$ and defined by

$$
p_{\lambda} f(x)=\arg \min _{z}\left\{f(z)+\frac{1}{2 \lambda}\|z-x\|^{2}\right\} .
$$

The following lemma shows that (13) can be expressed as a nonsmooth equation. As it is an obvious result from the definition of the proximal mapping, we omit its proof.

Lemma 4. Equation (13) is equivalent to the following nonsmooth equation:

$$
F(x)+\nabla\left[e_{\lambda} \phi\right](x-\lambda F(x))=0
$$

or

$$
x-\left[p_{\lambda} \phi\right](x-\lambda F(x))=0,
$$

for any $\lambda>0$.

Let

$$
G_{\lambda}(x)=x-\left[p_{\lambda} \phi\right](x-\lambda F(x)),
$$

which is named as KKT mapping for problem (1). Obviously, we have that the solution set for problem (1) is $G_{\lambda}^{-1}(0)$. Let $\bar{x} \epsilon$ $G_{\lambda}^{-1}(0)$. We discuss the isolated calmness of $G_{\lambda}$ at $(0, \bar{x})$.

From Lemma 3, we can easily obtain the following result.

Theorem 5. Assume that the proximal mapping $p_{\lambda} \phi$ is directionally differentiable. Then $G_{\lambda}$ is isolated clam at $(0, \bar{x})$ if and only if

$$
G_{\lambda}^{\prime}(\bar{x} ; d)=0 \Longrightarrow d=0 .
$$

In the following, we consider two cases of MVIP: in the first case $\phi(z)=\|z\|_{1}=\sum_{i=1}^{m}\left|z_{i}\right|$, and in the second case $\phi(z)=\delta_{p}(z)$, where $P$ is a convex polyhedral set. In particular, we consider the nonlinear complementarity problem, which corresponds to $P=R_{+}^{m}$ in the second case.

Case $1\left(\phi(z)=\|z\|_{1}=\sum_{i=1}^{m}\left|z_{i}\right|\right)$. We first introduce some notations. Let $\alpha=\left\{i: x_{i}^{0}>0\right\}, \beta=\left\{i: x_{i}^{0}=0\right\}$, and $\gamma=\{i$ : $\left.x_{i}^{0}<0\right\}$ be three index sets such that $\beta=\beta_{U} \cup \beta_{S} \cup \beta_{L}$, where

$$
\begin{aligned}
& \beta_{U}=\left\{i: x_{i}^{0}=0, F_{i}\left(x^{0}\right)=-1\right\}, \\
& \beta_{S}=\left\{i: x_{i}^{0}=0,-1<F_{i}\left(x^{0}\right)<1\right\}, \\
& \beta_{L}=\left\{i: x_{i}^{0}=0, F_{i}\left(x^{0}\right)=1\right\} .
\end{aligned}
$$

Let $T=\{1, \ldots, n\} \backslash \beta_{S}$. Define the critical cone at $x^{0}$ as follows:

$$
C\left(x^{0}\right)=\left\{\begin{array}{c}
d_{T}: 0 \leq d_{\beta_{U}} \perp J_{x_{T}} F_{\beta_{U}}\left(x^{0}\right) d_{T} \geq 0 \\
0 \geq d_{\beta_{L}} \perp J_{x_{T}} F_{\beta_{L}}\left(x^{0}\right) d_{T} \leq 0
\end{array}\right\} .
$$

We give a set of sufficient conditions for the isolated calmness of $G_{\lambda}$ at a point $\left(0, x^{0}\right)$, where $x^{0} \in G_{\lambda}^{-1}(0)$.

Proposition 6. Let $F: R^{n} \rightarrow R^{n}$ be a continuously differentiable mapping. Assume that $x^{0} \in R^{n}$ is a solution to problem (1) with $\phi(z)=\|z\|_{1}$ for $z \in R^{n}$. If for $\forall d_{T} \in$ $C\left(x^{0}\right) \backslash\{0\}$,

$$
\left\langle d_{T}, J_{x_{T}} F_{T}\left(x^{0}\right) d_{T}\right\rangle \neq 0,
$$

then $G_{\lambda}$ is isolated calm at $\left(0, x^{0}\right)$.

Proof. When $\phi(z)=\|z\|_{1}$, it is easy to obtain

$$
v \in \partial \phi\left(x^{0}\right) \Longleftrightarrow v_{i} \begin{cases}=1, & x_{i}^{0}>0, \\ \in[-1,1], & x_{i}^{0}=0, \\ =-1, & x_{i}^{0}<0 .\end{cases}
$$

Therefore, we obtain the following equivalence:

$$
\begin{gathered}
-F\left(x^{0}\right) \in \partial \phi\left(x^{0}\right) \Longleftrightarrow \\
F_{i}\left(x^{0}\right) \begin{cases}=-1, & x_{i}^{0}>0, \\
\in[-1,1], & x_{i}^{0}=0, \\
=1, & x_{i}^{0}<0 .\end{cases}
\end{gathered}
$$

We consider the following equation in variable $d$ :

$$
d-\left[p_{\lambda} \phi\right]^{\prime}\left(x^{0}-\lambda F\left(x^{0}\right) ; d-\lambda F^{\prime}\left(x^{0}\right) d\right)=0 .
$$

Nothing that $p_{\lambda} \phi(y)=[y-\lambda]_{+}+[y+\lambda]_{-}$, we have that (25) can be equivalently expressed as

$$
\begin{aligned}
d_{\beta_{S}} & =0, \\
J_{x_{T}} F_{\alpha \cup \gamma}\left(x^{0}\right) d_{T} & =0, \\
0 & \leq d_{\beta_{U}} \perp J_{x_{T}} F_{\beta_{U}}\left(x^{0}\right) d_{T}, \\
0 & \geq d_{\beta_{L}} \perp J_{x_{T}} F_{\beta_{L}}\left(x^{0}\right) d_{T} \leq 0 .
\end{aligned}
$$

This implies that $d_{T} \in C\left(x^{0}\right) \backslash\{0\}, d_{\beta_{S}}=0$, and $J_{x_{T}} F_{T}\left(x^{0}\right) d_{T}=$ 0 . We have from condition (22) that $d_{T}=0$. Thus, $G_{\lambda}^{\prime}(\bar{x} ; d)=$ 0 implies $d=0$ and from Theorem $5, G_{\lambda}$ is isolated calm at $\left(0, x^{0}\right)$. The proof is completed.

Case $2\left(\phi_{z}=\delta_{P}(z)\right.$ where $P$ is a convex polyhedral set). For a convex cone $K \in R^{n}$, its dual, denoted by $K^{*}$, is defined by

$$
K^{*}=\{w:\langle v, w\rangle \geq 0, \forall v \in K\} .
$$

For a point $x^{0} \in P$, where $P$ is a convex polyhedral set, the critical cone of the pair $(P, F)$ at $x_{0}$ is defined by

$$
C\left(x_{0}\right)=T_{P}\left(x_{0}\right) \cap F\left(x_{0}\right)^{\perp} .
$$


Proposition 7. Let $F: R^{n} \rightarrow R^{n}$ be a continuously differentiable mapping. Assume that $x^{0} \in R^{n}$ is a solution to problem (1) with $\phi(z)=\delta_{p}(z)$ for $z \in R^{n}$. Then $G_{\lambda}$ is isolated calm at $\left(0, x^{0}\right)$ if and only if

$$
C\left(x^{0}\right) \ni d \perp F^{\prime}\left(x^{0}\right) d \in C\left(x^{0}\right)^{*} \Longrightarrow d=0 .
$$

Proof. In this case, $\left(p_{\lambda} \phi\right)(y)=\prod_{P}(y)$, and $\partial \phi(y)=N p(y)$, so we have $-\lambda F\left(x^{0}\right) \in N p\left(x^{0}\right)$ for any $\lambda>0$. The equation $G_{\lambda}^{\prime}\left(x^{0} ; d\right)=0$ is

$$
d-\left[p_{\lambda} \phi\right]^{\prime}\left(x^{0}-\lambda f\left(x^{0}\right) ; d-\lambda F^{\prime}\left(x^{0}\right) d\right)=0,
$$

which is reduced to

$$
d-\prod_{P}^{\prime}\left(x^{0}-\lambda F\left(x^{0}\right) ; d-\lambda F^{\prime}\left(x^{0}\right) d\right)=0 .
$$

It follows from [17], for the directional derivative of the projection over a convex polyhedral set, that (31) is equivalent to

$$
d-\prod_{C\left(x^{0}\right)}\left(d-\lambda F^{\prime}\left(x^{0}\right) d\right)=0 .
$$

As $C\left(x^{0}\right)$ is a convex polyhedral cone, (32) is actually the following cone complementarity:

$$
C\left(x^{0}\right) \ni d \perp F^{\prime}\left(x^{0}\right) d \in C\left(x^{0}\right)^{*} .
$$

Using condition (29), we obtain from Theorem $5, G_{\lambda}$ is isolated calm at $\left(0, x^{0}\right)$.

Now we consider a special case when $P=R_{+}^{n}$. Define

$$
\begin{aligned}
& \alpha=\left\{i: x_{i}^{0}=0, F_{i}\left(x^{0}\right)>0\right\}, \\
& \beta=\left\{i: x_{i}^{0}=0=F_{i}\left(x^{0}\right)\right\}, \\
& \gamma=\left\{i: x^{0}>0, F_{i}\left(x^{0}\right)=0\right\} .
\end{aligned}
$$

Then we obtain the following result.

Corollary 8. The mapping $G_{\lambda}$ is isolated calm at $\left(0, x^{0}\right)$ if and only if

$$
\begin{gathered}
{\left[\begin{array}{cc}
J_{\beta} F_{\beta} & J_{\gamma} F_{\beta} \\
J_{\beta} F_{\gamma} & J_{\gamma} F_{\gamma}
\end{array}\right]\left[\begin{array}{c}
d_{\beta} \\
d_{\gamma}
\end{array}\right]} \\
\Downarrow\left[\begin{array}{c}
R_{+}^{|\beta|} \\
\{0\}
\end{array}\right] \\
\Downarrow \\
d_{\beta}=0, \\
d_{\gamma}=0 .
\end{gathered}
$$

Proof. Noting that, when $P=R_{+}^{n}$, we have

$$
\begin{aligned}
C\left(x^{0}\right) & =\left\{d: d \in T_{R_{+}^{n}}\left(x^{0}\right), d \perp F\left(x^{0}\right)\right\} \\
& =\left\{d: d_{\alpha}=0, d_{\beta} \geq 0\right\}, \\
C\left(x^{0}\right)^{*} & =\left\{d: d_{\beta} \geq 0, d_{\gamma}=0\right\} .
\end{aligned}
$$

We obtain the result from Proposition 7.

\section{VIP with Equality and \\ Inequality Constraints}

In this section, we consider the following variational inequality problem (for short, denoted by VIP): find $x \in K$ such that

$$
\langle F(x), y-x\rangle \geq 0, \quad \forall y \in K,
$$

where

$$
K=\left\{x \in R^{n}: h(x)=0, g(x) \leq 0\right\},
$$

with $h: R^{n} \rightarrow R^{p}$ and $g: R^{n} \rightarrow R^{p}$ being twice continuously differentiable.

Let $\bar{x}$ be a solution to VIP (37). We need the following assumption.

Assumption 9. The constraint set $K$ satisfies MangasarianFromovitz constraint qualification at $\bar{x}$, namely,

(i) $\nabla h_{1}(\bar{x}), \ldots, \nabla h_{q}(\bar{x})$ are linearly independent;

(ii) there exists a vector $d_{0} \in R^{n}$ such that

$$
\begin{aligned}
& \nabla h_{j}(\bar{x})^{T} d_{0}=0, \quad j=1, \ldots, q, \\
& \nabla g_{i}(\bar{x})^{T} d_{0}<0, \quad i \in I(\bar{x}),
\end{aligned}
$$

where $I(\bar{x})=\left\{i: g_{i}(\bar{x})=0, i=1, \ldots, p\right\}$.

Under Assumption 9, the normal cone of $K$ at $\bar{x}$ coincides with the regular normal cone, which is given by

$$
\begin{aligned}
N_{K}(\bar{x})= & \widehat{N}_{K}(\bar{x}) \\
= & \text { Range } J h(\bar{x})^{T} \\
& +\left\{J g(\bar{x})^{T} \lambda: 0 \leq \lambda \perp g(\bar{x})\right\} .
\end{aligned}
$$

From the definition of regular normal cone, if $\bar{x}$ is a solution to VIP (37), then we have

$$
0 \in F(\bar{x})+\widehat{N}_{K}(\bar{x}) .
$$

Therefore, we obtain from (40) that there exists $(\bar{\mu}, \bar{\lambda}) \in R^{q} \times$ $R^{p}$ such that

$$
\begin{aligned}
0 & =F(\bar{x})+J h(\bar{x})^{T} \bar{\mu}+J g(\bar{x})^{T} \bar{\lambda}, \\
h(\bar{x}) & =0, \\
0 & \leq \bar{\lambda} \perp g(\bar{x}) \leq 0 .
\end{aligned}
$$

System (42) is named as the KKT conditions for VIP (37). The set of all points $(\bar{x}, \bar{\mu}, \bar{\lambda})$ satisfying (42) is denoted by $\bar{\Omega}$.

Define

$$
G(x, \mu, \lambda)=\left[\begin{array}{c}
F(x)+J h(x)^{T} \mu+J g(x)^{T} \lambda \\
h(x)=0 \\
g(x)-\prod_{R_{-}^{p}}(g(x)+\lambda)
\end{array}\right] .
$$

Obviously, we have $G^{-1}(0)=\bar{\Omega}$. We discuss the isolated calmness of $G^{-1}$ at a point $(0,(\bar{x}, \bar{\mu}, \bar{\lambda}))$. In order to obtain our main results, we need the following lemmas. 
Lemma 10. Let $a \in R_{-}^{p}, b \in N_{R_{-}^{p}}(a), \Delta a$, and $\Delta b \in R^{p}$ satisfy

$$
\Delta a-\prod_{R_{-}^{p}}(a+b, \Delta a+\Delta b)=0 .
$$

Then

(i) $\Delta a \in T_{R^{p}}(a) \cap b^{\perp}$;

(ii) $\langle\Delta a, \Delta b\rangle=0$;

(iii) $\Delta b \in\left[T_{R_{-}^{p}}(a) \cap b^{-1}\right]^{o}$.

Proof. From Facchinei and Pang (2003), we have

$$
\prod_{R_{-}^{p}}(a+b, \Delta a+\Delta b)=\prod_{T_{R_{-}^{p(a) n b^{\perp}}}}(\Delta a+\Delta b) .
$$

Thus, we obtain from (44) that

$$
\Delta a=\prod_{T_{R P}(a) \cap b^{\perp}}(\Delta a+\Delta b),
$$

which implies

$$
T_{R_{-}^{p}}(a) \cap b^{\perp} \ni \Delta a \perp \Delta b \in\left[T_{R_{-}^{p}}(a) \cap b^{\perp}\right]^{o} .
$$

Thus, we get the properties (i), (ii), and (iii).

Lemma 11. Let $A_{1} \in R^{q \times n}, A_{2} \in R^{p \times n}, a \in R_{-}^{P}$, and $b \in$ $N_{R^{p}}(a)$ satisfy

$$
A R^{n}+T_{R_{-}^{p}}(a) \cap b^{\perp}=R^{q+p},
$$

where $A=\left[A_{1}, A_{2}\right]$, and then

$$
\begin{aligned}
A_{1}^{T} \Delta a+A_{2}^{T} \Delta b & =0, \\
\prod_{R_{-}^{P}}^{\prime}(a+b, \Delta b) & =0 \\
\Downarrow & \\
\Delta a & =0, \\
\Delta b & =0 .
\end{aligned}
$$

Proof. For $(\Delta a, \Delta b) \in\left[A R^{n}+T_{R_{-}^{p}}(a) \cap b^{\perp}\right]^{o}$, one has

$$
\begin{aligned}
A^{\mathrm{T}}[\Delta a, \Delta b] & =0, \quad \Delta b \in\left[T_{R_{-}^{p}}(a) \cap b^{\perp}\right]^{o}, \\
A_{1}^{\mathrm{T}} \Delta a+A_{2}^{\mathrm{T}} \Delta b & =0, \\
\prod_{R_{-}^{P} \cap b^{\perp}}(\Delta b) & =0 \Longleftrightarrow \prod_{R_{-}^{P}}(a+b, \Delta b)=0 .
\end{aligned}
$$

Thus, the conclusion comes from the polar of the left-side of (48) being a zero cone. The proof is completed.
Theorem 12. Assume that $F$ is continuously differentiable. Let $(\bar{x}, \bar{\mu}, \bar{\lambda}) \in R^{n} \times R^{q} \times R^{p}$. Consider the following conditions:
(i) $(\bar{x}, \bar{\mu}, \bar{\lambda}) \in G^{-1}(0) \neq \phi$.
(ii) For any $d \in C(\bar{x}) \backslash\{0\}$.

$$
\langle J L(\bar{x}, \bar{\mu}, \bar{\lambda}) d, d\rangle>0
$$
holds:

(iii) Strict Mangasarian-Fromovitz constraint qualification

$$
\begin{aligned}
R^{q+p}= & {\left[\begin{array}{c}
J h(\bar{x}) \\
J g(\bar{x}) \\
g(x)-\prod_{R_{-}^{p}}(g(x)+\lambda)
\end{array}\right] R^{n} } \\
& +T_{\left\{0_{q}\right\} \times R_{-}^{p}}(0, g(\bar{x})) \cap\left[\begin{array}{c}
\bar{\mu} \\
\bar{\lambda}
\end{array}\right]^{\perp} .
\end{aligned}
$$

If conditions (i), (ii), and (iii) are satisfied, then $G^{-1}$ is isolated calm at $(0,(\bar{x}, \bar{\mu}, \bar{\lambda}))$.

Proof. We only check the following implication:

$$
G^{\prime}(\bar{x}, \bar{\mu}, \bar{\lambda} ; d) \Longrightarrow d=0
$$

where $d=\left(d_{x}, d_{\mu}, d_{\lambda}\right) \in R^{n} \times R^{q} \times R^{p}$. By direct calculation, we obtain from (53) that

$$
\begin{array}{r}
L(\bar{x}, \bar{\mu}, \bar{\lambda}) d_{x}+J h(\bar{x})^{\mathrm{T}} d_{\mu}+J g(\bar{x})^{\mathrm{T}} d_{\lambda}=0, \\
J h(\bar{x}) d_{x}=0, \\
J g(\bar{x}) d_{x}-\prod_{R_{-}^{p}}^{\prime}\left(g(\bar{x})+\bar{\lambda}, J g(\bar{x}) d_{x}+d_{\lambda}\right)=0 .
\end{array}
$$

It follows from Lemma 10 that

$$
\begin{gathered}
J g(\bar{x}) d_{x} \in T_{R_{-}^{p}}(g(\bar{x})) \cap \bar{\lambda}^{\perp}, \\
d_{\bar{\lambda}} \in\left[T_{R_{-}^{p}}(g(\bar{x})) \cap \bar{\lambda}^{\perp}\right]^{o}, \\
\left\langle J g(\bar{x}) d, d_{\lambda}\right\rangle=0 .
\end{gathered}
$$

From KKT conditions for VIP (37), we have

$$
\begin{aligned}
F(\bar{x})^{\mathrm{T}} d_{x} & =\left[-J h(\bar{x})^{\mathrm{T}} \bar{\mu}-J g(\bar{x})^{\mathrm{T}} \bar{\lambda}\right]^{\mathrm{T}} d_{x} \\
& =-\left\langle\bar{\mu}, J h(\bar{x}) d_{x}\right\rangle-\left\langle\bar{\lambda}, J g(\bar{x}) d_{x}\right\rangle=0 .
\end{aligned}
$$

Combining the above properties about $d$, we have that $d_{x} \in$ $C(\bar{x})$. Then, by premultiplying $d_{x}^{\mathrm{T}}$ to the first line of (55), we get

$$
\left\langle d_{x}, J_{x} L(\bar{x}, \bar{\mu}, \bar{\lambda}) d_{x}\right\rangle=0,
$$

implying $d_{x}=0$ from (ii). 
Noticing $d_{x}=0$, we have from (55) that

$$
\begin{aligned}
J h(\bar{x})^{\mathrm{T}} d_{u}+J g(\bar{x})^{\mathrm{T}} d_{\lambda} & =0, \\
\prod_{R_{-}^{p}}^{\prime}\left(g(\bar{x})+\lambda, d_{\lambda}\right) & =0 .
\end{aligned}
$$

We simplify (iii) as

$$
\left[\begin{array}{l}
J h(\bar{x}) \\
J g(\bar{x})
\end{array}\right] R^{n}+\left[\begin{array}{c}
0 \\
T_{R_{-}^{p}}(g(\bar{x})) \cap \bar{\lambda}^{\perp}
\end{array}\right]=R^{q+p} .
$$

We obtain from Lemma 11 and (58) that $d_{\mu}=0, d_{\lambda}=0$, which completed the proof.

Remark 13. An interesting question is whether (i), (ii), and (iii) are necessary for the isolated calmness of $G^{-1}$ at $(0,(\bar{x}, \bar{\mu}, \bar{\lambda}))$. In the near future, we will pay attention to the study of this question.

\section{Conflicts of Interest}

The authors declare that there are no conflicts of interest regarding the publication of this paper.

\section{Acknowledgments}

This work is supported by the National Natural Science Foundation of China under Projects nos. 11371070 and 11371071.

\section{References}

[1] H. Brézis, Opérateurs Maximaux Monotones et Semi-Groupes de Contractions dans les Espaces de Hilbert, North-Holland, Amsterdam, The Netherlands, 1973.

[2] M. A. Noor, "A class of new iterative methods for general mixed variational inequalities," Mathematical and Computer Modelling, vol. 31, no. 13, pp. 11-19, 2000.

[3] R. T. Rockafellar, Convex Analysis, Princeton Mathematical Series, No. 28, Princeton University Press, Princeton, NJ, USA, 1970.

[4] D. Han and X. Yuan, "Local linear convergence of the alternating direction method of multipliers for quadratic programs," SIAM Journal on Numerical Analysis, vol. 51, no. 6, pp. 34463457, 2013.

[5] M. Hong and Z.-Q. Luo, "On the linear convergence of the alternating direction method of multipliers," Mathematical Programming, vol. 162, no. 1-2, Ser. A, pp. 165-199, 2017.

[6] D. R. Han, D. F. Sun, and L. W. Zhang, "Linear rate convergence of the alternating direction method of multipliers for convex composite quadratic and semi-definite programming," http://arxiv.org/abs/1508.02134, 2015.

[7] S. M. Robinson, "Strongly regular generalized equations," Mathematics of Operations Research, vol. 5, no. 1, pp. 43-62, 1980.

[8] H. T. Jongen, D. Klatte, and K. Tammer, "Implicit functions and sensitivity of stationary points," Mathematical Programming, vol. 49, no. 1, (Ser. A), pp. 123-138, 1990/91.

[9] A. L. Dontchev and R. T. Rockafellar, "Characterizations of strong regularity for variational inequalities over polyhedral convex sets," SIAM Journal on Optimization, vol. 6, no. 4, pp. 1087-1105, 1996.
[10] Y. Zhang and L. Zhang, "On the upper Lipschitz property of the KKT mapping for nonlinear semidefinite optimization," Operations Research Letters, vol. 44, no. 4, pp. 474-478, 2016.

[11] Y. J. Liu and S. H. Pan, "Locally upper Lipschitz of the perturbed KKT system of Ky Fan k-norm matrix conic optimization problems," http://arxiv.org/abs/1509.00681, 2015.

[12] C. Ding, D. Sun, and L. Zhang, "Characterization of the robust isolated calmness for a class of conic programming problems," SIAM Journal on Optimization, vol. 27, no. 1, pp. 67-90, 2017.

[13] B. S. Mordukhovich and M. E. Sarabi, "Critical multipliers in variational systems via second-order generalized differentiation," Mathematical Programming, pp. 1-44, 2017.

[14] R. T. Rockafellar and R. J.-B. Wets, Variational Analysis, Springer, New York, 1998.

[15] A. J. King and R. T. Rockafellar, "Sensitivity analysis for nonsmooth generalized equations," Mathematical Programming, vol. 55, no. 2, Ser. A, pp. 193-212, 1992.

[16] A. B. Levy, "Implicit multifunction theorems for the sensitivity analysis of variational conditions," Mathematical Programming, vol. 74, no. 3, Ser. A, pp. 333-350, 1996.

[17] F. Facchinei and J.-S. Pang, Finite-dimensional variational inequalities and complementarity problems. Vol. I, Springer Series in Operations Research, Springer-Verlag, NY, USA, 2003. 


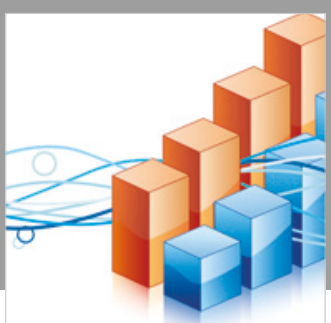

Advances in

Operations Research

\section{-n-m}
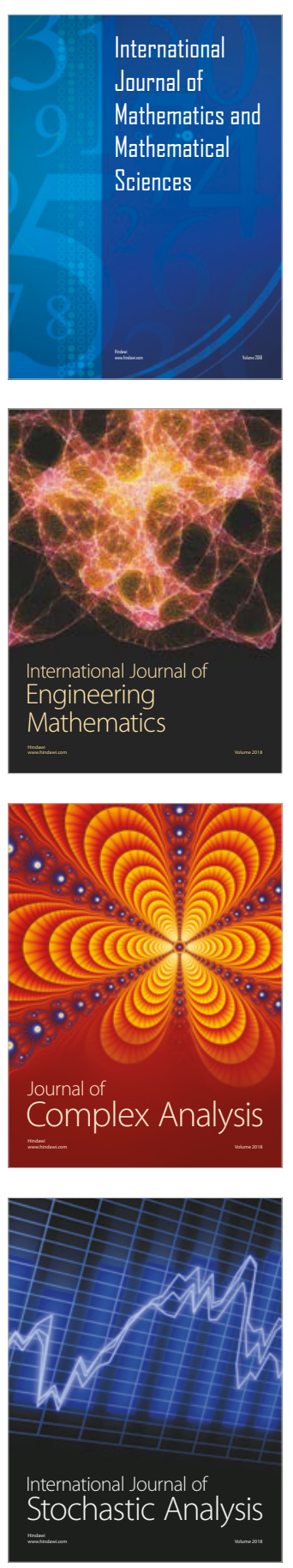
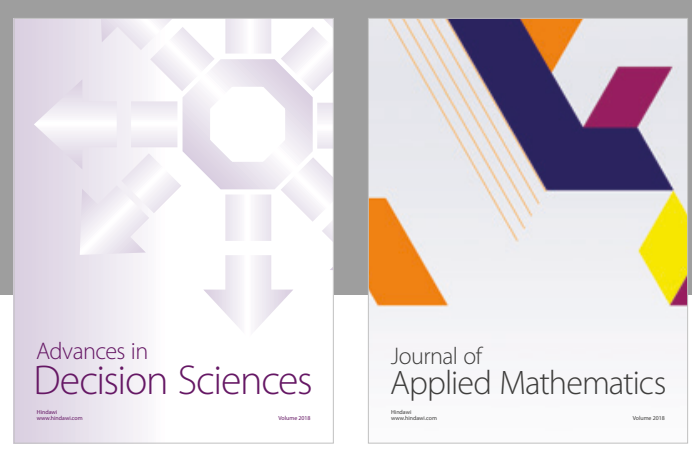

Journal of

Applied Mathematics
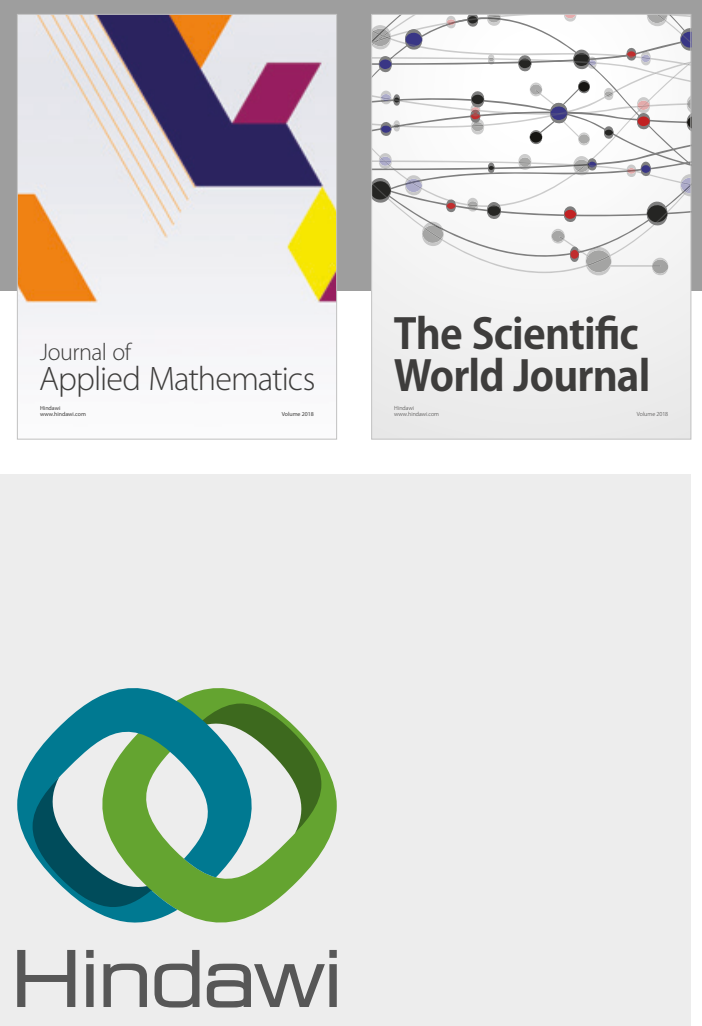

Submit your manuscripts at

www.hindawi.com

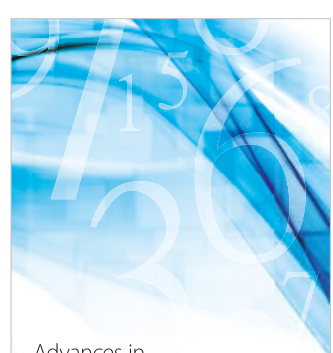

Advances in
Numerical Analysis
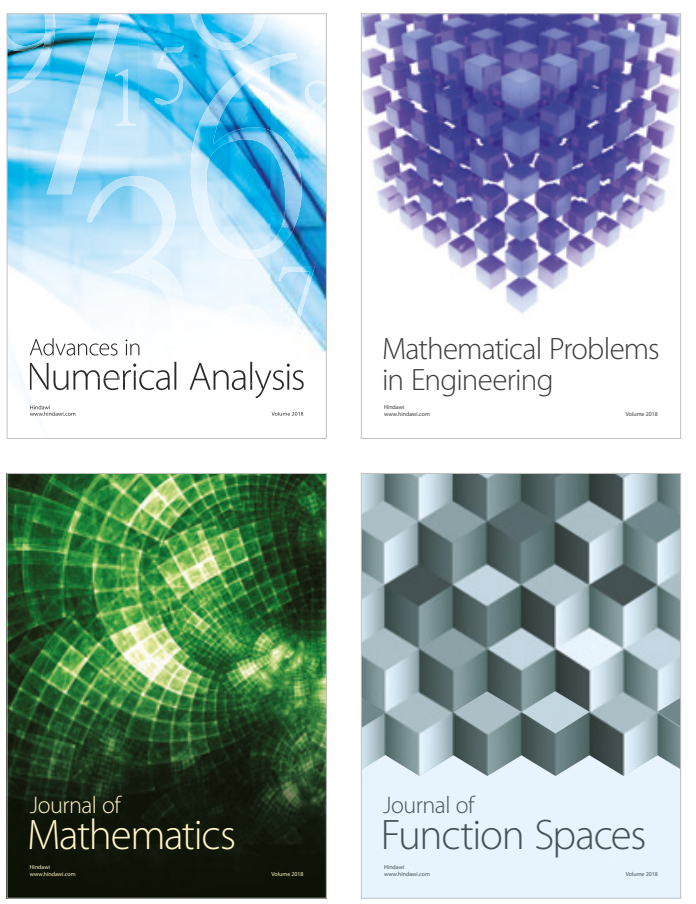

Mathematical Problems in Engineering

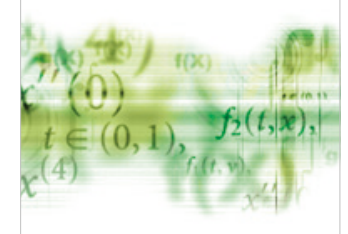

International Journal of

Differential Equations

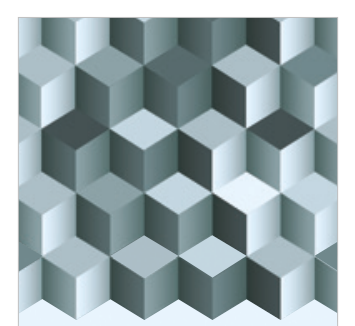

Journal of

Function Spaces

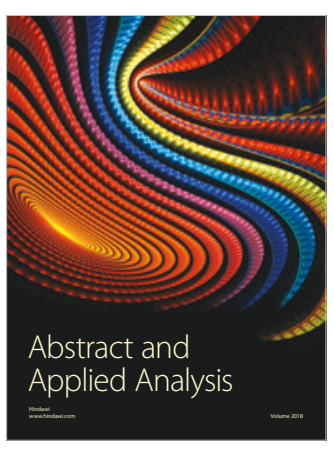

The Scientific

World Journal

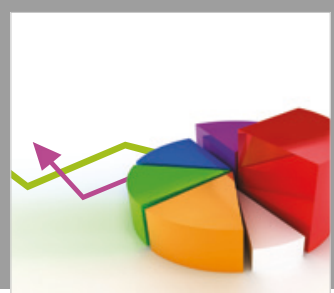

Journal of

Probability and Statistics
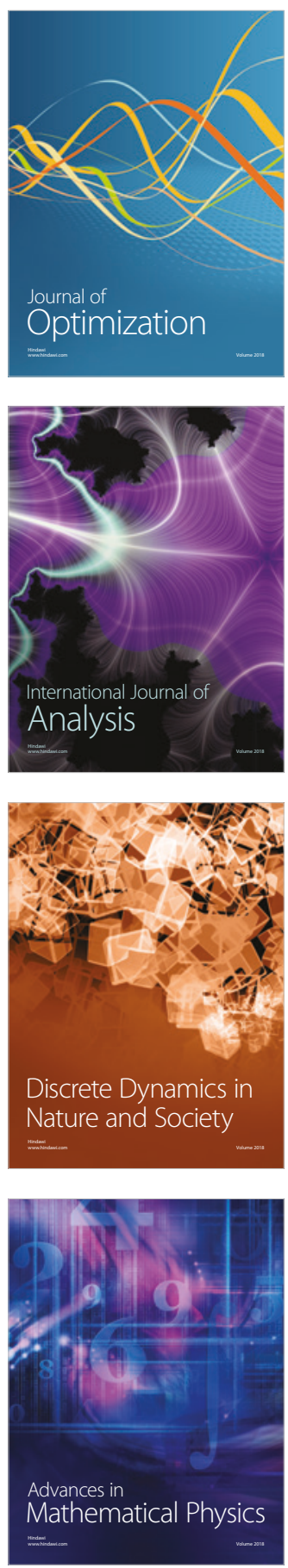